\title{
Permeability of dura mater: a possible link between cortical spreading depression and migraine pain? A comment
}

\author{
Peer Carsten Tfelt-Hansen
}

Received: 18 July 2010/Accepted: 16 August 2010/Published online: 7 September 2010

(C) Springer-Verlag 2010

\begin{abstract}
In the wake of cortical spreading depression (CSD) it has been suggested that noxious substances diffuse through the dura with resulting firing of epidural nerves. In my view this is unlikely because there are good reasons to suggest that there must be a dura-brain barrier.Alternatively collateral branches from the trigeminal nerve to the pia and the dura may signal what is happening with ions and substances on the brain surface during CSD to the epidural space.
\end{abstract}

Recently it was stated in a paper [1] on cortical spreading depression (CSD) and activation of meningeal nociceptors in rats: "The going theory is that the hyperemic phase of CSD is associated with local release of ATP and glutamate and potassium and hydrogen ions by neurons, glia, or vascular cells. Regardless of the origin of their release, these mediators are thought to diffuse outward into the leptomeninges overlaying the affected cortical region, resulting in activation of pial nociceptors, local neurogenic inflammation and the persistent activation of dural nociceptors which triggers the migraine headache" [1]. The latter postulates that the dura mater is permeable for these noxious molecules. This is, however, unlikely since it would create problems with the blood-brain barrier (BBB) system. The epidural capillaries and veins have no $\mathrm{BBB}$ and the extracellular epidural space have the same content of ions and substances as the blood. If there was no dura-brain barrier then this would circumvent the BBB.

P. C. Tfelt-Hansen $(\bowtie)$

Department of Neurology, Danish Headache Center, University of Copenhagen, Glostrup Hospital, Glostrup, Denmark

e-mail: ptha@glo.regionh.dk
For example, the concentration of glutamate is $100 \mu \mathrm{mol} / \mathrm{l}$ in the blood whereas it is only $1-4 \mu \mathrm{mol} / 1$ in the extracellular fluid in the brain [2-4]. The very low extracellular glutamate concentrations is essential for normal brain function [3], and if there was no barrier glutamate would tend to diffuse into the brain with deleterious effects. This would be a teleological catastrophe.

What could be the components of this postulated durabrain barrier? The dura itself could be impermeable for purely physical reasons and the subdural arachnoidea could be an active barrier like the BBB. Furthermore, it could be a combination of both.

Whatever the details it is most likely that there is an impermeable dura-brain barrier and that ions such as glutamate released from the pial surface cannot diffuse through the dura and activate epidural nociceptors.

An alternative and more plausible possibility for activation of epidural nociceptors is axon reflexes within ramifications of trigeminal axons both to the pia and the dura mater [5]. Such collateral branches have been demonstrated in the mouse [6] and it has been demonstrated in rats that CSD causes a dilatation of and increased blood flow in the middle meningeal artery in a trigeminal parasympaticus-dependent manner [5]. In addition, immediate short-lived burst of firing by meningeal nociceptors was observed after single CSD and as suggested by the authors this immediate short-lived burst of firing is probably mediated by axonal collaterals that extended from the dura into the pia [1]. In other trials, however, an immediate or delayed long-lasting activation of dural nociceptors was observed [1] and the mechanism of this activation is less clear.

The possible existence of collateral branches of the trigeminal nerve to the pia and dura should be investigated further in man. 


\section{References}

1. Zhang XC, Levy D, Noseda R, Kainz V, Jekuboski M, Burstein R (2010) Activation of meningeal nocicsptors by cortical spreading depression: implications for migraine with aura. J Neurosci 30:8007-8814

2. Baker DA, Xi Z-X, Shen H, Swanson CD, Kalivas PW (2002) The origin and neuronal function of in vivo nonsynaptic glutamate. J Neurosci 22:9134-9141

3. Hawkins RA (2009) The blood-brain barrier and glutamate. Am J Clin Nutr 90:867S-874S
4. Kilpatrick M, Church E, Danish S, Stiefel M, Jaggi J, Halpern C, Maloney E, Robinson M, Lucki I, Krisman-Grenda E, Batuch G (2010) Intracerebral microdialysis during deep brain stimulation surgery. J Neurosci Methods 190:106-111

5. Bolay H, Reuter U, Dunn AK, Huang Z, Boas DA, Moskowitz MA (2002) Intrinsic braine activity triggers trigeminal meningeal afferents in a migraine model. Nat Med 8:136-142

6. Kosaras B, Jakubowski M, Kainz V, Burstein R (2009) Sensory innervation of the calvarial bones of the mouse. J Comp Neurol 515:331-348 